\title{
PRACI - Perceção dos utentes sobre antibioterapia, Resistência a Antimicrobianos e Controlo de Infeção
}

Nuno Miguel Parente*

\section{RESUMO}

Objetivo: Caracterizar os conhecimentos dos utentes sobre práticas inerentes à antibioterapia, concetualização de resistência a antimicrobianos (RAB), controlo de infeção e averiguar a existência de relação entre a informação sobre RAB e restantes variáveis.

Tipo de estudo: Estudo observacional, transversal e analítico.

Local: USF Viver Mais.

População: Utentes inscritos na USF Viver Mais com 18-69 anos ( $n=6.952)$.

Métodos: Questionário anónimo aplicado a uma amostra de 365 utentes. Incluiu variáveis demográficas como idade, sexo e escolaridade, presentes na Parte I, e 15 perguntas de escolha simples na Parte II sobre conceito e funcionamento do antimicrobiano $(A B)$, atitudes relativas ao $A B$, adequação do $A B$ a determinados sintomas e doenças, práticas de controlo de infeção, fonte de informação sobre RAB, definição e dimensão da RAB.

Resultados: Houve maior representatividade do sexo feminino (61,9\%), do grupo com $45-54$ anos e escolaridade $\geq 12^{\circ}$ ano. $A$ maioria reconheceu o papel dos $A B$ nas infeções víricas e mais de $70 \%$ identificou os $A B$ como fármacos anti-piréticos e anti-inflamatórios. A principal indicação para $A B$ foi a odinofagia com duração $\geq 5$ dias e aproximadamente $89 \%$ concordou em utilizar $A B$ de familiares/amigos. Cerca de $50 \%$ considerou importante a higienização das mãos após contacto com uma instituição de saúde e $69,4 \%$ após contacto com secreções respiratórias. O reconhecimento do conceito da RAB constatou-se em $58,1 \%$ dos utentes, em que $50 \%$ a percecionou como problema pessoal e familiar. Mais de $88 \%$ conceptualizou a RAB como uma falha de resposta do corpo aos $\mathrm{AB}$.

Verificou-se relação entre a informação sobre RAB do doente e as seguintes variáveis: escolaridade; conhecimento sobre o efeito negativo do $A B$ na flora autóctone; importância do $A B$ no tratamento da constipação, gripe e odinofagia; autossuspeição do utente da necessidade de tratamento com $A B$; decisão de antibioterapia gerida pelo médico e confiança no médico não-prescritor de $A B(p<0,05)$.

Conclusões: Em geral, os utentes demonstraram baixo conhecimento nas diversas áreas, apesar de sobreponível à literatura. Deste modo, o médico de família pode dirigir as suas ações para a informação adequada do conceito de RAB e implicações no ecossistema.

Palavras-chave: Resistência a antimicrobianos; Antibióticos; Controlo de infeção; Literacia em saúde.

\section{INTRODUÇÃO}

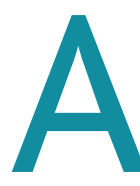
tualmente, a resistência aos antimicrobianos (RAB) é um dos problemas de saúde pública mais preocupantes a nível global, com ameaça de regresso à era pré-antibiótico. ${ }^{1} \mathrm{Na} \mathrm{Eu}-$ ropa, o impacto das infeções por bactérias resistentes reflete-se anualmente em aproximadamente 33.110 mortes e 874.541 anos de vida ajustados para incapacidade. $^{2}$ Relativamente ao custo, na União Europeia

*Médico interno do $4^{\circ}$ ano da formação específica em Medicina Geral e Familiar. USF Viver Mais, ACeS Maia/Valongo 
sabe-se que a infeção por bactérias resistentes leva a gastos anuais na ordem dos 1,5 biliões de euros em despesas de saúde e perda de produtividade. ${ }^{3}$ A variação do nível de RAB é ampla e dependente da estirpe bacteriana, uso local de antimicrobianos $(\mathrm{AB})$ e região geográfica; contudo, é transversal o seu aumento desmedido. ${ }^{4}$ À imagem da Europa, Portugal apresenta como problema principal as bactérias do grupo ESKAPE (Enterococcus faecium, Staphylococus aureus, Klebsiella pneumoniae, Acinectobacter baumannii, Pseudomonas aeruginosa e Enterobacter spp.) e enterobacteriáceas produtoras de $ß$-lactamases de largo espectro ou carbapenemases. ${ }^{5}$ De acordo com a Rede de Vigilância Europeia de Resistência aos Antimicrobianos, de 2016 para 2017 obteve-se uma evolução favorável do nível de resistência aos diferentes grupos de $A B$, à exceção do aumento da resistência da $E$. Coli aos carbapenemos e da resistência global da $K$. pneumoniae. ${ }^{6} \mathrm{O}$ contínuo aumento da RAB à escala mundial, na ausência de abordagem síncrona e eficaz face a este problema, prevê 10 milhões de mortes/ano associadas a infeções por bactérias resistentes e gastos na ordem dos 60-100 triliões de dólares até $2050 .^{7}$

O consumo de $\mathrm{AB}$ de forma inadequada é o fator modificável e mais frequentemente implicado na indução de RAB. ${ }^{8}$ Em Portugal, o consumo de AB na comunidade, expresso em dose diária definida por 1.000 habitantes por dia, aumentou de 19,6 para 20,3 entre 2013 e $2017 . .^{9}$

De acordo com as orientações do Programa Nacional de Prevenção e Controlo de Infeções e Resistências aos Antimicrobianos, a estratégia global de intervenção visa a informação e educação da população com dinamização de campanhas de sensibilização para o uso prudente dos AB. São exemplos a colaboração com o Ministério da Educação no projecto $e$-Bug e futuramente na implementação do projecto PORCAUSA, dirigido à literacia da população. ${ }^{10}$ De facto, o uso inadequado de $\mathrm{AB}$ depende de fatores intrínsecos e extrínsecos ao profissional de saúde, dos quais se salientam o conhecimento, atitude e comportamento do cidadão, o perfil prescritor, a subvalorização da existência de RAB e a vontade de corresponder às expectativas do doente e/ou família. ${ }^{6,11-13} \mathrm{Um}$ aspeto fundamental é que a sobreprescrição de $\mathrm{AB}$ leva o doente a requerer um comportamento de prescrição semelhante numa con- sulta futura, perpetuando o ideal de necessidade falsa de tratamento com $\mathrm{AB} .{ }^{14}$ É igualmente transversal que o conhecimento da doença e opções terapêuticas, crenças sobre o balanço benefício-dano das mesmas e expectativas relativas ao resultado, são variáveis que influenciam a adesão e, por conseguinte, o sucesso do tratamento. ${ }^{15}$

Tendo por base que na Europa $80-90 \%$ dos $A B$ são prescritos nos cuidados de saúde primários (CSP), ${ }^{16} \mathrm{O}$ autor pretende com este trabalho caracterizar os conhecimentos sobre práticas inerentes à antibioterapia, concetualização de RAB e controlo de infeção numa população dos CSP e averiguar a existência de relação entre a informação sobre RAB e as restantes variáveis.

\section{MÉTODOS}

Foi conduzido um estudo transversal, descritivo e analítico, entre 1 de agosto de 2018 e 31 de janeiro de 2019, numa Unidade de Saúde Familiar (USF) da zona norte de Portugal.

O autor concebeu um questionário de autopreenchimento e adaptado de bibliografia já publicada, ${ }^{17-22}$ anónimo, que foi aplicado após estudo piloto. O questionário incluiu variáveis demográficas: idade (variável qualitativa ordinal), sexo (variável qualitativa nominal) e escolaridade (variável qualitativa ordinal), presentes na Parte I; e quinze perguntas de escolha simples na Parte II (Anexo I). Nesta última secção, as perguntas referiam-se à perceção do uso de $\mathrm{AB}$ (indicações, reações adversas, condições de acesso, adequação a determinadas doenças/sintomas e decisão de iniciação do regime terapêutico), de medidas de controlo de infeção e de RAB (existência, definição, fonte de informação, dimensão problemática, indução e prevenção).

A população incluiu utentes com idade entre 18-69 anos, inscrição ativa na USF em estudo e consulta agendada no período de recolha de dados. Foram excluídos utentes com agendamento de consulta de saúde infantil e visita domiciliária; os utentes incapazes de compreender linguagem portuguesa, oral e escrita; e os que não preencheram o questionário na totalidade. A população em estudo totalizou 6.952 utentes. Foi selecionada uma amostra de conveniência com base nos utentes que, aquando da efetivação da inscrição para consulta, foram convidados a participar no estudo pelo secretariado clínico, o qual teve uma sessão de formação 


\begin{tabular}{|l|r|}
\hline \multicolumn{2}{|l|}{ QUADRO I. Dados demográficos } \\
\hline Sexo & $\boldsymbol{n}(\%)$ \\
\hline Masculino & $139(38,1)$ \\
Feminino & $226(61,9)$ \\
\hline Idade & \\
$18-24$ anos & $30(8,2)$ \\
$25-34$ anos & $54(14,8)$ \\
$35-44$ anos & $73(20,0)$ \\
$45-54$ anos & $86(23,6)$ \\
$55-64$ anos & $72(19,7)$ \\
$65-69$ anos & $50(13,7)$ \\
\hline Escolaridade & $76(20,8)$ \\
$1-4^{\circ}$ ano & $37(10,1)$ \\
$5-6^{\circ}$ ano & $81(22,2)$ \\
$7-9^{\circ}$ ano & $85(23,3)$ \\
$10-11^{\circ}$ ano & $86(23,6)$ \\
$\geq 12^{\circ}$ ano & 365 \\
\hline Total & \\
\hline
\end{tabular}

Legenda: $\%$ = percentagem.

prévia com o autor, de forma a uniformizar o guião do convite. No caso de aceitação foi entregue o questionário que incluía, em preâmbulo, a explicação do propósito do estudo e o consentimento informado. Os questionários foram recolhidos em caixa fechada e selada para o efeito.

O tamanho amostral foi determinado considerando um intervalo de confiança de $95 \%$ e um nível de precisão de 5\%, perfazendo um total de 364 participantes. Considerando o ajuste para perda de amostra de aproximadamente $20 \%$, assumiu-se 455 participantes como tamanho amostral final.

Os dados foram submetidos a uma análise descritiva com recurso aos programas Microsoft Excel $2010^{\circledR} \mathrm{e}$ SPSS $^{\circledR}$ v. (64 Bit) 21 e avaliação de associação entre as variáveis categóricas, utilizando o teste do Qui-quadrado.

Foram utilizadas as linhas de orientação Strengthening the Reporting of Observational Studies in Epidemiology (STROBE) para a elaboração do presente artigo.
A realização deste estudo foi aprovada pelo ACeS Maia/Valongo e mereceu parecer favorável por parte da comissão de ética da Administração Regional de Saúde do Norte.

\section{RESULTADOS}

Foram incluídos 365 utentes, o que resultou numa taxa de participação de $80,2 \%$. A população teve um predomínio do sexo feminino $(61,9 \%)$, da faixa etária dos $45-54$ anos (23,6\%) e escolaridade igual ou superior ao $12^{\circ}$ ano (23,6\%) (Quadro I).

Relativamente à questão número 1 , a maioria dos participantes completou o último curso de $\mathrm{AB}$ há mais de 12 meses $(34,2 \%)$ e $31,2 \%$ não se recordou da última toma. Na questão número $2,80 \%$ da população afirmou que o $A B$ é útil no tratamento de infeções causadas por bactérias; contudo, 56,7\% referiu também indicação do mesmo para o tratamento de infeções víricas. Uma proporção considerável dos utentes $(73,2-74,8 \%)$ considerou o $\mathrm{AB}$ um medicamento semelhante à classe dos anti-inflamatórios e útil na resolução de quadros de febre. Em relação à questão número 3, entre 75,3 e 78,9\% dos utentes consideraram que $\mathrm{o} A \mathrm{~B}$ tem efeitos adversos e pode dar origem a reações alérgicas graves. No entanto, 40,5\% não atribuíram ao $\mathrm{AB}$ a capacidade de «matar bactérias boas presentes no corpo». No que concerne à questão número 4 foi averiguado o perfil de condições de acesso aos AB (Figura 1). Na questão número 5 foi avaliada a perceção do utente quanto à utilização do $\mathrm{AB}$ como tratamento primário de determinados sintomas e doenças (Figura 2).

Na questão número 6, 50,4\% dos doentes assumiu ter capacidade para suspeitar do momento em que é necessário tratamento com $\mathrm{AB}$. Perante a possibilidade de decidir e iniciar antibioterapia por iniciativa própria, $82,7 \%$ respondeu «não sei» e 12,6\% tê-lo-á feito no passado. Porém, 95,3\% demonstrou que a decisão para início de antibioterapia deve partir do médico. No que concerne à relação médico-doente, $87,9 \%$ revelou ter confiança no médico prescritor de $\mathrm{AB}$, enquanto $73,2 \%$ mantém a confiança se a atitude não resultar em prescrição antibiótica.

Na questão número 7 verificou-se que foram reconhecidas, como medidas protetoras contra a contaminação por microorganismos, a lavagem das mãos em $93,7 \%$ e a ventilação natural em $68,8 \%$. Para além disso, 


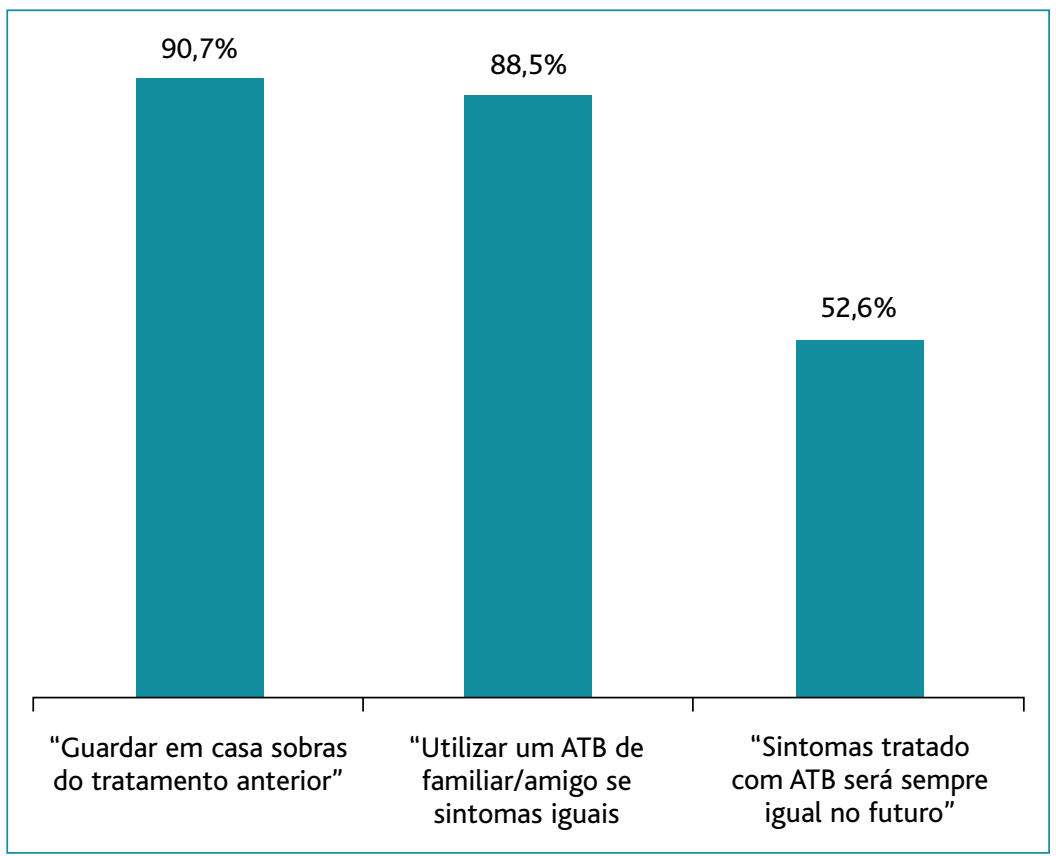

Figura 1. Práticas no uso do ATB.

Legenda: $\mathrm{ATB}=$ antibiótico; $\%$ = percentagem.

abandono de qualquer instituição de saúde, $50,1 \%$ e $38,4 \%$ concorda e discorda, respetivamente, com esta prática.

Relativamente à questão número 8 , $58,1 \%$ dos doentes demonstraram-se informados sobre a existência de RAB. A caracterização da fonte de informação foi avaliada na questão número 9 (Figura 3). Na questão número 10 , referente à concetualização da RAB, 88,7\% concordaram que assenta no facto de o corpo não responder a determinado $\mathrm{AB}$ e $56,6 \%$ que se deve à perda da sensibilidade da bactéria a um ou mais AB. Trinta e três por cento afirmaram que as bactérias portadoras de RAB são transmissíveis de pessoa para pessoa e $62,3 \%$ reconheceram que as infeções causadas por estas bactérias são mais difíceis de tratar. Na questão número 11 verificou-se que $86,8 \%$ dos doentes não recusaram/adiaram a toma de algum $\mathrm{AB}$ por medo relacionado com a RAB.

Na questão número $12,68,9 \%$ declarou que a RAB é um problema mundial e causa de morte cada vez mais frequente. Relativamente à ocorrência da RAB de forma restrita a nível hospitalar, $6,6 \%$ concordaram com a afirmação e $84,4 \%$ responderam «não sei». Aproximadamente $50 \%$ dos doentes admitiram a RAB como um problema afeto ao seu Figura 2. Adequação de $A B$ a sintomas e doenças. Legenda: \% = percentagem.

92,9\% concordou que a lavagem das mãos é obrigatória após contacto com secreções respiratórias e 71,8\% concordou que o braço serve de proteção perante acesso de tosse. Relativamente à lavagem das mãos antes do ambiente familiar. Na questão número 13, referente a ações benéficas a tomar no combate ao aumento da $\mathrm{RAB}, 25,7 \%$ referiu a diminuição do uso de herbicidas na agricultura, $25,4 \%$ o maior rigor no uso de $\mathrm{AB}$ pelo 


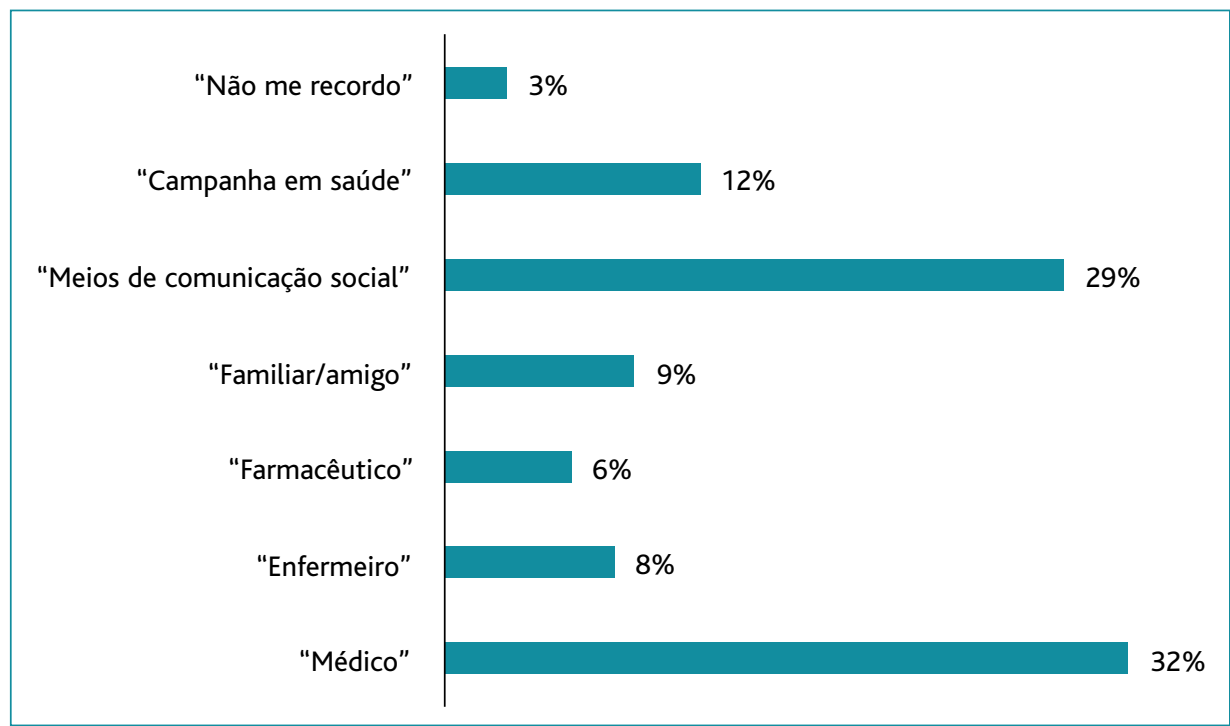

Figura 3. Fonte de informação sobre RAB.

Legenda: $\mathrm{RAB}=$ resistência a antimicrobianos; \% = percentagem.

veterinário e 23,5\% a criação de novos $A B$. Na questão $14,64,2 \%$ dos doentes afirmou que a RAB resulta do uso incorreto do $\mathrm{AB}$ e $42,0 \%$ revelou que o $\mathrm{AB}$ utilizado de forma desnecessária numa determinada região pode ser prejudicial para os seus residentes. Para além disto, 83,5\% considerou o cumprimento das indicações médicas como fundamentais à prevenção da RAB, enquanto $48,1 \%$ valorizou o papel da prescrição tardia.

Verificou-se relação entre a informação sobre RAB do doente e as seguintes variáveis: escolaridade; conhecimento sobre o efeito negativo do $\mathrm{AB}$ na flora autóctone; importância do $\mathrm{AB}$ no tratamento da constipação, gripe e odinofagia; autossuspeição do utente da necessidade de tratamento com AB; decisão de antibioterapia gerida pelo médico e confiança no médico não-prescritor de AB (Quadro II).

\section{DISCUSSÃO}

De uma forma global, os resultados mostram um conhecimento deficitário no que diz respeito à antibioterapia, RAB e controlo de infeção. Comparando com a literatura verifica-se que, apesar de $40 \%$ da população reconhecer os vírus como agentes primários de infeções das vias aéreas superiores, ${ }^{23} 47,8 \%$ acredita que o $\mathrm{AB}$ tem papel no tratamento. ${ }^{18}$ Este último valor é inferior ao encontrado $(56,7 \%)$ e poderá ser explicado pela população estudada ser holandesa e, portanto, detentora de um maior nível de literacia em saúde e menor taxa de prescrição antibiótica. ${ }^{24}$ Este padrão de desconhecimento é dependente da região estudada e, por isso, na Malásia a proporção de doentes que identificam o $\mathrm{AB}$ como tratamento de infeções víricas pode ascender aos $83,7 \% .{ }^{25}$ É também interessante um estudo realizado numa farmácia comunitária da Malásia, com 408 utentes, em que $43,1 \%$ identifica erradamente o paracetamol como AB, 65,9\% identifica erradamente a aspirina como pertencente à nova geração de $A B$ e $46,6 \%$ acredita que o $A B$ funciona como anti-pirético. ${ }^{26}$ Estes valores mais baixos relativamente ao encontrado $(73,2-74,8 \%)$ podem dever-se ao facto de $96,8 \%$ dos utentes terem, de escolaridade, pelo menos o ensino secundário. ${ }^{26}$

Os doentes veem o $\mathrm{AB}$ como uma opção muito segura, ${ }^{27}$ contudo, neste estudo a maioria reconhece o $\mathrm{AB}$ como fonte de efeitos adversos e possíveis reações alérgicas graves. Na Itália, um estudo com 1.247 pais de crianças entre 0-14 anos demonstrou que 92,6-94,8\% acredita que os $\mathrm{AB}$ são causadores de efeitos adversos e reações alérgicas. ${ }^{19}$ Estes números assemelham-se aos obtidos; contudo, o facto de a amostra ser exclusivamente constituída por pais pode acarretar sobrevalorização. Ainda referente aos efeitos adversos dos AB, um estudo transversal no Reino Unido, com 7.120 indivíduos, verificou que $43 \%$ desconhecem o efeito do $A B$ na flora da pele e intestino e, portanto, semelhante ao encontrado (40\%). ${ }^{28} \mathrm{~A}$ clarificação e discussão deste efeito e dos anteriores são fundamentais na modelação da expectativa e adoção de atitude mais reticente pelo doente. ${ }^{23,29}$

No que concerne o acesso ao $\mathrm{AB}$, dois estudos, na Suécia $(n=747)$ e no Japão $(n=3.390)$, demonstraram, respetivamente, que $7,6 \%$ e $11,7 \%$ dos utentes admitem guardar excedente de $\mathrm{AB}$ em casa. ${ }^{21,30}$ Porém, o valor encontrado $(90,7 \%)$ é claramente superior e assemelha-se 


\section{QUADRO II. Fatores relacionados com a informação sobre RAB}

Informado sobre a RAB

\begin{tabular}{|c|c|c|c|}
\hline \multirow[b]{2}{*}{ Escolaridade } & Sim & Não & \multirow[b]{2}{*}{$p<0,0001^{*}$} \\
\hline & & & \\
\hline $1-4^{\circ}$ ano & 11 & 65 & \\
\hline $5-6^{\circ}$ ano & 18 & 19 & \\
\hline $7-9^{\circ}$ ano & 49 & 32 & \\
\hline $10-11^{\circ}$ ano & 61 & 24 & \\
\hline$\geq 12^{\circ}$ ano & 73 & 13 & \\
\hline "Pode matar bactérias boas presentes no nosso corpo" & & & $p<0,0001^{*}$ \\
\hline Concordo & 127 & 47 & \\
\hline Discordo & 68 & 80 & \\
\hline Não sei & 17 & 26 & \\
\hline Sintomas/doenças & & & $p<0,0001^{*}$ \\
\hline \multicolumn{4}{|l|}{ Constipação } \\
\hline Sim & 10 & 25 & \\
\hline Não & 202 & 128 & \\
\hline \multicolumn{4}{|l|}{ Gripe } \\
\hline Sim & 59 & 70 & \\
\hline Não & 153 & 83 & \\
\hline \multicolumn{4}{|l|}{ Dor de garganta com mais de 5 dias } \\
\hline Sim & 103 & 124 & \\
\hline Não & 98 & 40 & \\
\hline $\begin{array}{l}\text { "Normalmente sou capaz de suspeitar quando preciso } \\
\text { de tratamento com AB" }\end{array}$ & & & $p<0,001^{*}$ \\
\hline Concordo & 91 & 93 & \\
\hline Discordo & 23 & 19 & \\
\hline Não sei & 98 & 41 & \\
\hline "A decisão de iniciar AB deve ser tomada pelo médico" & & & $p<0,009^{*}$ \\
\hline Concordo & 203 & 133 & \\
\hline Discordo & 4 & 9 & \\
\hline Não sei & 5 & 11 & \\
\hline "Tenho confiança no médico se não receitar AB" & & & $p<0,0001^{*}$ \\
\hline Concordo & 180 & 87 & \\
\hline Discordo & 19 & 64 & \\
\hline Não sei & 13 & 2 & \\
\hline
\end{tabular}

Legenda: $\mathrm{RAB}=$ resistência a antimicrobianos; $p=$ nível de significância; ${ }^{*}=$ estatisticamente significativo. a outros países, como Brasil $(84,1 \%)$ ou China $(90,0 \%) .{ }^{31}$ É importante esclarecer que a população sueca, no campo das práticas com $\mathrm{AB}$, tem um conhecimento elevado e consistente ao longo do tempo ${ }^{32}$ e que no estudo japonês mais de $96,6 \%$ tinha escolaridade $\geq 10^{\circ}$ ano, pertencendo unicamente $7,5 \%$ a um meio rural. Este resultado superior pode significar que a amostra se enquadra nas ideologias de reutilização do $\mathrm{AB}$, presente em $50 \%$ dos doentes, e vontade de reclassificação do $\mathrm{AB}$ para medicamento não sujeito a receita médica. ${ }^{31}$ De facto, este excedente de $\mathrm{AB}$ pode resultar da sobreprescrição e/ou de menor adesão terapêutica. A falta de adesão correlaciona-se com o número de tomas/dia do $\mathrm{AB}(27,0 \%$ se $\geq 3)$, duração do curso $(71,0 \%$ se $\geq 6$ dias), menor qualidade do serviço médico e personalidade do doente (crítico). ${ }^{33}$ Para além disto, são também influentes a política nacional de dispensa do $\mathrm{AB}$, nomeadamente, a necessidade de prescrição médica, o fornecimento de embalagem completa ${ }^{34} \mathrm{e}$ a disponibilização de informação sobre a indicação e posologia do $\mathrm{AB}$ que podem reduzir em $14,2 \%$ o risco de guardar $\mathrm{AB}$ excedente. ${ }^{31}$ Dos $\mathrm{AB}$ não sujeitos a prescrição médica, a proporção com origem em familiares e amigos, dependendo da região europeia, pode variar entre $32-81 \% .{ }^{35}$ Este cenário permite enquadrar o valor encontrado $(88,5 \%)$ e teorizar no modo como os doentes valorizam o autodiagnóstico. Efetivamente, em 24,9$37,4 \%$ há a crença de que um sintoma/doença tratado com $\mathrm{AB}$ 
num determinado momento será submetido ao mesmo tratamento no futuro, sugerindo a perceção de «inevitabilidade». ${ }^{23}$ Esta perceção pode advir das propriedades atribuídas ao $\mathrm{AB}$ e, deste modo, um estudo qualitativo com 5.379 doentes mostrou que em $51-87 \%$ o $\mathrm{AB}$ é associado a ideias de «cura certa», "potência elevada» e «estatuto de salvador». ${ }^{36}$

Neste estudo, $10 \%$ e $35 \%$ da população revelou a constipação e gripe, respetivamente, como indicações principais para tratamento com $\mathrm{AB}$. Na literatura a distinção entre estas duas entidades é imprecisa semanticamente; contudo, os valores são semelhantes e indicam que $18,9-49,7 \%^{12,21,23,26,37-38}$ dos doentes julgam o binómio constipação/gripe como razão para prescrição de AB. Especificamente, até $30,2 \%$ caracteriza a prescrição de $\mathrm{AB}$ na gripe como «boa atitude». ${ }^{21}$ Relativamente às outras possíveis indicações para $\mathrm{AB}$, um estudo na Arábia Saudita ( $n=1.966)$ reconheceu igualmente a odinofagia $(48,8 \%)$ como prevalente, ${ }^{37}$ enquanto outro trabalho aponta também a bronquite aguda $(59,6 \%)$ e a pneumonia $(90,9 \%)$ como indicações reportadas. ${ }^{18}$ Por oposição, a odinofagia surge com mais peso na amostra, o que está em concordância com um estudo português em que a amigdalite foi o principal motivo para toma de $\mathrm{AB}$ entre 282 indivíduos. ${ }^{38}$

Existe uma «necessidade» de prescrição com $A B$ por parte do doente sendo que, no Qatar, $28,1 \%$ espera tratamento com $\mathrm{ATB}^{23}$ e, no Japão, 10,2\% formaliza um pedido por consulta. ${ }^{30} \mathrm{Um}$ estudo transversal na Holanda, com 935 participantes, obteve resultados semelhantes. Cerca de 37\% admitem capacidade de suspeição para tratamento com $\mathrm{AB}$ e 92,9\% acreditam que a decisão de iniciar $\mathrm{AB}$ deve ser tomada pelo médico, valores inferiores aos encontrados, 50,4\% e 95,7\%, respetivamente. ${ }^{18}$ Neste estudo é esta elevada percentagem de transferência da decisão para o médico que inibe provavelmente a promoção do autodiagnóstico e, portanto, resulta em 12,6\% de automedicação com AB. Na maioria dos casos, esta «carência» cega transportada no $\mathrm{AB}$ assenta nos mitos de recuperação acelerada e prevenção de agravamento do quadro. ${ }^{12}$ Além disso, em situações agudas, $49,6 \%$ transmite insatisfação com ausência de prescrição de $A B$ e $31,6 \%$ procura essa prescrição noutra instituição. ${ }^{23}$ No que diz respeito à confiança depositada no médico, em função de o resultado ser a presença/ausência de prescrição de $A B$, um estu- do referido previamente demonstrou maior confiança no médico com menor volume de prescrição antibiótica.$^{30}$ Este resultado é contrário ao verificado na amostra e noutro trabalho ${ }^{32}$ e pode espelhar a dificuldade da população portuguesa na modificação de atitudes em relação ao $\mathrm{AB}$, constatando-se ainda ideologias «embrionárias» e falsas e, consequentemente, também um determinado grau de insatisfação para com o médico não-prescritor. Relativamente à automedicação, o valor encontrado está de acordo com outros estudos em Portugal $(4,0-17,1 \%) .{ }^{37,39} \mathrm{Na}$ Europa pode variar até os $21 \%$ em países do Leste, relacionando-se de forma direta com a idade jovem, escolaridade superior e presença de doença crónica. ${ }^{40}$ Num contexto de países em desenvolvimento, a prevalência de automedicação com $\mathrm{AB}$ pode ascender aos $38,8 \%$, maioritariamente com fonte em farmácias comunitárias e associada a baixa condição socioeconómica, experiência prévia positiva e severidade dos sintomas. ${ }^{41}$

No que concerne a práticas de controlo de infeção, um estudo na Coreia com 877 participantes demonstrou que $56,9 \%$ utilizam o braço como proteção perante um acesso de tosse e $69,4 \%$ compreende a lavagem das mãos como importante após contacto com secreções respiratórias, valores inferiores aos encontrados no estudo, respetivamente, $71,8 \%$ e $92,9 \% .{ }^{42}$ A valorização da lavagem das mãos antes de abandonar uma instituição de saúde para a prevenção de infeções futuras reportou-se a $50 \%$ dos doentes e vem reforçar o facto de só $10 \%$ utilizar efetivamente a higienização das mãos. ${ }^{43}$ Contudo, mais de $76 \%$ dos utentes consideraram importante a política de higienização das mãos na escolha do hospital ao qual recorrer e tinham interesse em aprender mais sobre este tópico. ${ }^{44}$ Isto pode significar que os utentes compreendem a higienização das mãos como um comportamento essencial, têm disponibilidade para aprofundar o conhecimento, mas ainda é definido como hospitalo-cêntrico e aparentemente sem impacto na incidência de infeções futuras e hetero-proteção.

No que concerne ao reconhecimento do conceito de RAB, o valor encontrado $(58,1 \%)$ é sobreponível a um estudo prévio $(59,4 \%)^{11}$ e a fonte de informação mais citada é o médico. ${ }^{30}$ No estudo sobressai o poder da comunicação social e das campanhas em saúde. Estes resultados são paradoxais, visto afirmarem uma maior 
predisposição da população para informação em saúde através de profissionais de saúde e iniciativas de órgãos médicos, em detrimento de outras fontes; porém, é ainda possível atentar à supremacia dos meios de comunicação social, mais frequentemente com transmissão de informação não cuidada.

No que diz respeito ao mecanismo de RAB, a literatura mostra-nos que $84,7-88 \%$ dos doentes acreditam que se deve à ausência de resposta do nosso corpo ao $\mathrm{AB}^{21,45}$ e 59,4\% que advém perda da sensibilidade natural pela bactéria, deste modo, valores sobreponíveis aos encontrados. ${ }^{25}$ São também concordantes as proporções dos doentes que percecionam a dificuldade no tratamento das bactérias resistentes e sua possibilidade de transmissão, respetivamente, $68 \%$ e $27,1 \%$ em comparação com $62,3 \%$ e $33,0 \%$ na amostra. ${ }^{25,45}$

Relativamente à perceção da dimensão problemática inerente à RAB, no Reino Unido $79 \%$ dos doentes reconhecem a sua presença nos cuidados de saúde hospitalares. ${ }^{28} \mathrm{Na}$ amostra $6,6 \%$ assumiu esta realidade; no entanto, 84,6\% afirmou não ter informação sobre o assunto. Isto pode significar que apesar de haver a noção de RAB a nível hospitalar, a capacidade de a população perceber a migração de doentes e, consequentemente, das bactérias entre diferentes níveis de cuidados de saúde é baixa. Um estudo numa clínica de dermatologia, com 512 participantes, demonstrou resultados superiores em que 50,7\% acredita que a RAB é «um dos maiores problemas que o Mundo enfrenta» e 86,3\% que é um «assunto que pode afetar a família». ${ }^{46}$ No entanto, $73,5 \%$ tinham escolaridade equivalente ao ensino superior, o que pode explicar a maior sensibilização para o possível impacto da RAB na família. O valor obtido foi superior na apreciação da RAB como «problema mundial» e pode dever-se ao papel mais preponderante dos meios de comunicação social na maior compreensão da RAB como problema grave de saúde pública. Contudo, é fundamental relevar que os meios de comunicação social levam a desinformação relativamente à RAB e que pode ser agravada com a frequência de utilização, criação e partilha pessoais de conteúdos e interação com mensagens de fatalismo. ${ }^{47}$

No campo das ações com benefício para a RAB, a maioria responde corretamente. Porém, 18,8\% consideraram a atualização do plano nacional de vacinação e $6,6 \%$ a interrupção do tratamento $\mathrm{AB}$, aquando da melhoria, como duas medidas potencialmente favoráveis. Um estudo em Vizela ${ }^{38}$ comprovou que $84 \%$ completa o tratamento com $\mathrm{AB}$ o que poderá explicar a baixa incidência da medida relacionada com a interrupção, que é também sobreponível a outros estudos. ${ }^{19,21}$

Em relação à indução e prevenção de RAB, 60,6\% e $70 \%$ dos doentes em dois trabalhos diferentes mostram que estes acreditam que a RAB advém do uso incorreto de $\mathrm{AB},,^{20,45}$ o que se assemelha à proporção de $64,2 \%$ encontrada. Entre $82-96,4 \%$ dos doentes cumprem as indicações posológicas do médico relativamente à toma do $\mathrm{AB}$, o que está de acordo com o valor encontrado $(83,5 \%)$, apesar de ser difícil concluir que compreendem a medida como preventiva de RAB. ${ }^{25,37} \mathrm{O}$ papel das prescrições tardias como prevenção de RAB foi aceite por $48,1 \%$ da amostra e apresenta-se concordante com a literatura $(37,4 \%) .{ }^{18}$ Cinquenta e oito por cento não reconheceu o efeito de propagação regional da $\mathrm{RAB}$ e realmente parece haver uma perceção pessoal de risco baixo quanto à $\mathrm{RAB}$, assumindo risco elevado em caso de internamento e tratamento prolongado com AB. ${ }^{45}$

Dois estudos transversais portugueses estudaram algumas das ideias relacionadas com práticas de antibioterapia. ${ }^{38-39}$ Contrariamente, este estudo acrescenta valor no campo da perceção da RAB e controlo de infeção.

Como limitações deste estudo apontar a técnica de amostragem por conveniência, subjacente a maior exequibilidade e gestão limitada de recursos, mas que pode ter incluído utentes hiperfrequentadores dos CSP e excluído utentes mais utilizadores do setor médico privado. Considerar também a ausência de estudo de validação do questionário para a população portuguesa, apesar do teste piloto e da base bibliográfica, e a constituição da amostra quanto ao predomínio dos jovens, distribuição do sexo e escolaridade que não permite validar externamente os dados.

Como pontos fortes focar o tamanho amostral, significativamente representativo da população da USF entre os 18-69 anos, o questionário baseado na literatura e o facto de o convite partir do secretariado clínico e não da equipa médica.

A maioria dos estudos estabelece um sistema de pontuação global para o questionário utilizado e avalia a associação entre essa pontuação e diferentes 
variáveis. ${ }^{18-19,21-22,28,32}$ Há evidência de que uma maior perceção sobre o conceito de RAB tem associação com fonte de informação médica, menor interrupção de tratamento com $\mathrm{AB}$ e melhor conhecimento das suas indicações. ${ }^{37}$ Para além do acima referido, neste estudo o conhecimento do conceito de RAB pelos utentes associou-se significativamente à escolaridade $\geq 7^{\circ}$ ano, à maior perceção do efeito nocivo do $\mathrm{AB}$ na flora autóctone, à melhor informação quanto ao papel do $\mathrm{AB}$ no tratamento da constipação, gripe e odinofagia, ao menor autodiagnóstico de necessidade de $\mathrm{AB}$ em determinado momento, à maior delegação na decisão de iniciar antibioterapia para o médico e à maior confiança no médico não-prescritor de $\mathrm{AB}$.

Este estudo, segundo o conhecimento do autor, é pioneiro na obtenção de informação proveniente dos utentes relativamente à RAB e controlo de infeção, para além de aprofundar também as questões das atitudes e crenças para com o AB nos CSP em Portugal. O âmbito do estudo foi a população entre os 18 e 69 anos, portanto, a aplicabilidade dos dados terá de ser dirigida a estas faixas etárias, considerando-se ainda relevante a predominância de jovens na amostra. É possível constatar que mesmo existindo um bom nível de escolaridade, $46,9 \%$ dos utentes tinham completado pelo menos o $10^{\circ}$ ano (dos quais $23,6 \%$ detinham a atual escolaridade obrigatória), o conhecimento sobre antibioterapia, RAB e controlo de infeção foi insuficiente. Contudo, é exatamente na população jovem que deve haver investimento formativo para evitar o agravamento da RAB. O estudo mostra que um utente jovem e informado relativamente à RAB está mais frequentemente apto a perceber o impacto negativo da toma de $A B$ no seu organismo e o quão infrutífera é a atividade do $\mathrm{AB}$ nas infeções predominantemente víricas. Para além disso, pode ainda compreender-se que esta informação sobre a RAB pode influenciar a predisposição do utente para autossuspeição da necessidade de $\mathrm{AB}$, possivelmente através da desmistificação de alguns conceitos, permitindo de igual forma uma maior racionalização do uso do $\mathrm{AB}$, através da delegação da decisão para o médico e aceitando mais facilmente o comportamento de não-prescrição, sem afetação da confiança na relação médico-doente. No entanto, é fulcral integrar a consciencialização sobre a RAB como um fenómeno bidirecional e cuja relação com o comporta- mento não é proporcional. Deste modo, profissionais de saúde com elevada perceção da RAB podem não restringir o consumo de $\mathrm{AB}$, mas dispensar mais frequentemente opções de $2^{\mathrm{a}}$ linha e ter contextos de desconhecimento do padrão local de resistências aos AB. ${ }^{48}$ Por outro lado, utentes participantes em sessões de educação para a saúde podem manter as atitudes prévias porque a experiência pessoal, a incerteza depositada no sistema de saúde e o aconselhamento dos familiares são, por vezes, fatores dominantes. ${ }^{49}$

\section{CONCLUSÃO}

O conhecimento desta população dos CSP relativamente a práticas de antibioterapia, RAB e controlo de infeção revelou-se baixo e sobreponível a estudos internacionais. De realçar a elevada proporção de doentes com facilidade em dispor de $\mathrm{AB}$ através de familiares/amigos e ferramentas para um autodiagnóstico de «necessidade» de $\mathrm{AB}$ com exercício de eventual pressão no médico. Apesar de não ser possível atribuir causalidade, é essencial referir a preponderância da comunicação social como fonte de informação preferencial dos utentes, frequentemente difusora de mensagem com teor alarmista e a ausência de noção na população da importância da higienização das mãos após contacto com uma instituição de saúde, provavelmente por autoperceção de baixo risco de RAB. É fundamental a maior promoção de atualização científica nesta área, por exemplo, através dos grupos coordenadores locais do Programa de Prevenção e Controlo de Infeções e de Resistência aos Antimicrobianos de cada ACeS, visando a capacitação dos médicos e enfermeiros de família neste campo. Assim sendo, é fulcral o médico de família, em sincronia com os outros profissionais de saúde dos CSP, dirigir as atividades de informação para a população jovem relativamente ao conceito de RAB e suas implicações no ecossistema, dado até $84 \%$ dos doentes querer discutir este tópico ${ }^{45} \mathrm{e}$ a desinformação estar associada a ideologias falsas que podem constituir perigo para a saúde. De acordo com o relatório de 2018 do Programa de Prevenção e Controlo de Infeções e de Resistência aos Antimicrobianos da Direção-Geral da Saúde, em julho de 2019 iniciar-se-á a campanha PORCAUSA com o objetivo de promover a utilização segura do $\mathrm{AB}$ na população nacional. ${ }^{10} \mathrm{Em}$ consonância com este projeto poder-se-ão planear ações de educação ao 


\section{nível dos CSP, visando a mudança de comportamento e trabalhando em conjunto na literacia da população.}

\section{AGRADECIMENTOS}

XXXX

A participação de todos foi indispensável.

\section{REFERÊNCIAS BIBLIOGRÁFICAS}

1. World Health Organization. Antimicrobial resistance: global report on surveillance, 2014 [Internet]. Geneva: WHO; 2014. ISBN 9789241564748. Available from: https://www.who.int/drugresistance/documents/surveillancereport/en/

2. Cassini A, Högberg LD, Plachouras D, Quattrocchi A, Hoxha A, Simonsen GS, et al.Attributable deaths and disability-adjusted life-years caused by infections with antibiotic-resistant bacteria in the EU and the European Economic Area in 2015: a population-level modelling analysis. Lancet Infect Dis. 2019;19(1):56-66.

3. European Centre for Disease Prevention and Control. The bacterial challenge: time to react [Internet]. Stockholm: ECDC; 2009. ISBN 9789291931934. Available from: https://ecdc.europa.eu/sites/portal/files/media/en/publications/Publications/0909_TER_The_Bacterial_Challenge_Time_to_React.pdf

4. Cars O, Mölstad S, Melander A. Variation in antibiotic use in the European Union. Lancet. 2001;357(9271):1851-3.

5. Loureiro RJ, Roque F, Rodrigues AT, Herdeiro MT, Ramalheira E. O uso de $A B$ e as resistências bacterianas: breves notas sobre a sua evolução [Use of antibiotics and bacterial resistances: brief notes on its evolution]. Rev Port Saúde Pública. 2016;34(1):77-84. Portuguese

6. European Centre for Disease Prevention and Control. Surveillance of antimicrobial resistance in Europe: annual report of the European Antimicrobial Resistance Surveillance Network (EARS-Net) 2017 [Internet]. Stockholm: ECDC; 2018. ISBN 9789294982797. Available from: https://ecdc.europa.eu/en/publications-data/surveillance-antimicrobial-resistance-europe-2017

7. O'Neill J, editor. Review on antimicrobial resistance: tackling a crisis for the health and wealth of nations [Internet]. London: HM Government; 2014. Available from: https://amr-review.org/sites/default/files/ AMR\%20Review\%20Paper\%20-\%20Tackling\%20a\%20crisis\%20for\% 20the\%20health\%20and\%20wealth\%20of\%20nations_1.pdf

8. Costelloe C, Metcalfe C, Lovering A, Mant D, Hay AD. Effect of antibiotic prescribing in primary care on antimicrobial resistance in individual patients: systematic review and meta-analysis. BMJ. 2010;340:c2096.

9. European Centre for Disease Prevention and Control. Antimicrobial consumption: annual epidemiological report for 2017 [Internet]. Stockholm: ECDC; 2018. Available from: https://www.ecdc.europa.eu/en/publications-data/antimicrobial-consumption-annual-epidemiologicalreport-2017

10. Direção-Geral da Saúde. Infeções e resistências aos antimicrobianos: relatório anual do programa prioritário 2018 [Internet]. Lisboa: Direção-Geral da Saúde; 2018. Available from: https://www.dgs.pt/portalda-estatistica-da-saude/diretorio-de-informacao/diretorio-de-informacao/por-serie-1003038-pdf.aspx?v=11736b14-73e6-4b34-a8e8d22502108547
11. Gualano MR, Gili R, Scaioli G, Bert F, Siliquini R. General population's knowledge and attitudes about antibiotics: a systematic review and meta-analysis. Pharmacoepidemiol Drug Saf. 2015;24(1):2-10.

12. Wood F, Phillips C, Brookes-Howell L, Hood K, Verheij T, Coenen S, et al. Primary care clinicians' perceptions of antibiotic resistance: a multi-country qualitative interview study. J Antimicrob Chemother. 2013;68(1):237-43.

13. Arroll B, Goodyear-Smith F, Thomas DR, Kerse N. Delayed antibiotic prescriptions: what are the experiences and attitudes of physicians and patients? J Fam Pract. 2002;51(11):954-9.

14. Little P, Gould C, Williamson I. Reattendance and complications in a randomised trial of prescribing strategies for sore throat: the medicalising effect of prescribing antibiotics. BMJ. 1997;315(7104):350-2.

15. Jackson C, Eliasson L, Barber N, Weinman J. Applying COM-B to medication adherence: a suggested framework for research and interventions. Eur Health Psychol. 2014;16(1):7-17.

16. Goossens H, Ferech M, Vander Stichele R, Elseviers M. Outpatient antibiotic use in Europe and association with resistance: a cross-national database study. Lancet. 2005;365(9459):579-87.

17. World Health Organization. Antibiotic resistance: multi-country public awareness survey [Internet]. Geneva: WHO; 2015. ISBN 9789241509817. Available from: https://www.who.int/drugresistance/documents/baselinesurveynov2015/en/

18. Cals JW, Boumans D, Lardinois RJ, Gonzales R, Hopstaken RM, Butler $\mathrm{CC}$, et al. Public beliefs on antibiotics and respiratory tract infections: an internet-based questionnaire study. Br J Gen Pract. 2007;57(545): 942-7.

19. Bert F, Gualano MR, Gili R, Scaioli G, Lovato E, Angelillo IF et al. Knowledge and attitudes towards the use of antibiotics in the paediatric age group: a multicenter survey in Italy. Eur J Public Health. 2017;27(3):50612.

20. Topor G, Grosu IA, Ghiciuc CM, Strat AL, Lupu oru CE. Awareness about antibiotic resistance in a self-medication user group from Eastern Romania: a pilot study. PeerJ. 2017;5:e3803.

21. André M, Vernby A, Berg J, Lundborg CS. A survey of public knowledge and awareness related to antibiotic use and resistance in Sweden. J Antimicrob Chemother. 2010;65(6):1292-6.

22. You JH, Yau B, Choi KC, Chau CT, Huang QR, Lee SS. Public knowledge, attitudes and behavior on antibiotic use: a telephone survey in Hong Kong. Infection. 2008;36(2):153-7.

23. Shaikhan F, Rawaf S, Majeed A, Hassounah S. Knowledge, attitude, perception and practice regarding antimicrobial use in upper respiratory tract infections in Qatar: a systematic review. JRSM Open. 2018;9(9): 2054270418774971

24. Sørensen K, Pelikan JM, Röthlin F, Ganahl K, Slonska Z, Doyle G, et al. Health literacy in Europe: comparative results of the European health literacy survey (HLS-EU). Eur J Public Health. 2015;25(6):1053-8.

25. Halim NA, Chang CT, Chan HK, Hassali MA, Nouri A. Knowledge and attitudes concerning antibiotic use and resistance among the public in Pulau Pinang, Malaysia. Malays J Med Sci. 2018;25(6):141-7.

26. Ling OA, Hassali MA, Al-Haddad MS, Syed Sulaiman SA, Shafie AA, Awaisu A. Public knowledge and attitudes towards antibiotic usage: a cross-sectional study among the general public in the state of Penang, Malaysia. J Infect Dev Ctries. 2011;5(5):338-47. 
27. Lum EP, Page K, Nissen L, Doust J, Graves N. Australian consumer perspectives, attitudes and behaviours on antibiotic use and antibiotic resistance: a qualitative study with implications for public health policy and practice. BMC Public Health. 2017;17(1):799.

28. McNulty CA, Boyle P, Nichols T, Clappison P, Davey P. Don't wear me out: the public's knowledge of and attitudes to antibiotic use. J Antimicrob Chemother. 2007;59(4):727-38.

29. Dekker AR, de Groot E, Sebalj T, Yardley L, Cals JW, Verheij TJ, van der Velden AW. Parents' attitudes and views regarding antibiotics in the management of respiratory tract infections in children: a qualitative study of the influence of an information booklet. BJGP Open. 2018;2(2):bjgpopen 18X101553.

30. Kamata K, Tokuda Y, Gu Y, Ohmagari N, Yanagihara K. Public knowledge and perception about antimicrobials and antimicrobial resistance in Japan: a national questionnaire survey in 2017. PLoS One. 2018;13 (11):e0207017.

31. Kardas P, Pechère JC, Hughes DA, Cornaglia G. A global survey of antibiotic leftovers in the outpatient setting. Int J Antimicrob Agents. 2007;30(6):530-6.

32. Vallin M, Polyzoi M, Marrone G, Rosales-Klintz S, Tegmark Wisell K, Stålsby Lundborg C. Knowledge and attitudes towards antibiotic use and resistance: a latent class analysis of a Swedish population-based sample. PLoS One. 2016;11(4):e0152160.

33. Pechère JC, Hughes D, Kardas P, Cornaglia G. Non-compliance with antibiotic therapy for acute community infections: a global survey. Int J Antimicrob Agents. 2007;29(3):245-53.

34. Lescure D, Paget J, Schellevis F, van Dijk L. Determinants of self-medication with antibiotics in European and Anglo-Saxon countries: a systematic review of the literature. Front Public Health. 2018;6:370.

35. Morgan DJ, Okeke IN, Laxminarayan R, Perencevich EN, Weisenberg S. Non-prescription antimicrobial use worldwide: a systematic review. Lancet Infect Dis. 2011;11(9):692-701.

36. Pechère JC. Patients' interviews and misuse of antibiotics. Clin Infect Dis. 2001;33 Suppl 3:S170-3.

37. Al-Shibani N, Hamed A, Labban N, Al-Kattan R, Al-Otaibi H, Alfadda S. Knowledge, attitude and practice of antibiotic use and misuse among adults in Riyadh, Saudi Arabia. Saudi Med J. 2017;38(10):1038-44.

38. Ribeiro M, Pinto I, Pedrosa C. Comportamento da população do concelho de Vizela no consumo de antibióticos [Antibiotics comsumption behaviour of the Vizela's region population]. Rev Port Saude Publica. 2009;27(2):57-70. Portuguese

39. Lopes HL, Pereira JB, Carvalho MR. O que sabem os utentes sobre antibióticos: um estudo de investigação em duas Unidades de Saúde Familiar [What patients know about antibiotics: a cross-sectional study in two Family Health Units]. Rev Port Med Geral Fam. 2015;31(4):24854. Portuguese

40. Grigoryan L, Haaijer-Ruskamp FM, Burgerhof JG, Mechtler R, Deschepper R, Tambic-Andrasevic A, et al. Self-medication with antimicrobial drugs in Europe. Emerg Infect Dis. 2006;12(3):452-9.

41. Ocan M, Obuku EA, Bwanga F, Akena D, Richard S, Ogwal-Okeng J, et al. Household antimicrobial self-medication: a systematic review and meta-analysis of the burden, risk factors and outcomes in developing countries. BMC Public Health. 2015;15:742.

42. Choi JS, Kim KM. Predictors of respiratory hygiene/cough etiquette in a large community in Korea: a descriptive study. Am J Infect Control. 2016;44(11):e271-3.

43. Birnbach DJ, Nevo I, Barnes S, Fitzpatrick M, Rosen LF, Everett-Thomas $\mathrm{R}$, et al. Do hospital visitors wash their hands? Assessing the use of alcohol-based hand sanitizer in a hospital lobby. Am J Infect Control. 2012;40(4):340-3.

44. Wu KS, Lee SS, Chen JK, Tsai HC, Li CH, Chao HL, et al. Hand hygiene among patients: attitudes, perceptions, and willingness to participate. Am J Infect Control. 2013;41(4):327-31.

45. McCullough AR, Parekh S, Rathbone J, Del Mar CB, Hoffmann TC. A systematic review of the public's knowledge and beliefs about antibiotic resistance. J Antimicrob Chemother. 2016;71(1):27-33.

46. Goettsche LS, Weig EA, Chung J, Hoff BM, Ince D, Wanat KA. Patient perceptions of antibiotic use and resistance at a single university dermatology clinic. J Dermatolog Treat. 2019;30(1):92-5.

47. Groshek J, Katz JE, Andersen B, Cutino C, Zhong Q. Media use and antimicrobial resistance misinformation and misuse: survey evidence of information channels and fatalism in augmenting a global health threat. Cogent Med. 2018;5:1460898.

48. Pearson $M$, Chandler C. Knowing antimicrobial resistance in practice: a multi-country qualitative study with human and animal healthcare professionals. Glob Health Action. 2019;12(1):1599560.

49. Haenssgen MJ, Xayavong T, Charoenboon N, Warapikuptanun P, Khine Zaw Y. The consequences of AMR education and awareness raising: outputs, outcomes, and behavioural impacts of an antibiotic-related educational activity in Lao PDR. Antibiotics (Basel). 2018;7(4):95.

\section{CONFLITO DE INTERESSES}

O autor declara não ter quaisquer conflitos de interesse.

\section{COMISSÃO DE ÉTICA}

Estudo realizado após parecer favorável da Comissão de Ética para a Saúde da ARS Norte.

\section{ENDEREÇO PARA CORRESPONDÊNCIA \\ Nuno Miguel Parente \\ E-mail: nunomiguelpm@gmail.com \\ https://orcid.org/0000-0002-4198-243X}

Recebido em 14-06-2019

Aceite para publicação em 11-08-2019 


\section{ABSTRACT}

PARIC - PATIENTS' PERCEPTION ON ANTIBIOTIC USE, ANTIMICROBIAL RESISTANCE AND INFECTION CONTROL Objective: To characterize the knowledge about practices inherent to antibiotics, the conceptualization of antimicrobial drug resistance (ADR), infection control and to ascertain the existence of a relationship between the information on ADR and remaining variables.

Type of study: Observational, transversal and analytical.

Location: USF Viver Mais.

Population: Patients enrolled in USF Viver Mais with 18-69 years $(n=6,952)$.

Methods: Anonymous questionnaire applied to a sample of 365 patients. It included demographic variables as age, sex, and schooling, present in Part I, and 15 simple choice questions in Part II.

Results: There was greater representation of the female sex (61.9\%), the group with $45-54$ years and schooling $\geq 12$ th year. Most recognized the role of antimicrobials $(A B)$ in viral infections and more than $70 \%$ identified $A B$ as antipyretic and anti-inflammatory drugs. The primary indication for $A B$ was sore throat with a duration $\geq 5$ days and nearly $89 \%$ agreed to use $A B$ from family/friends. About $50 \%$ considered it important to hand hygiene after contact with a health institution and $69.4 \%$ after contact with respiratory secretions. The recognition of ADR was verified in $58.1 \%$ of the patients, in which $50 \%$ perceived it as a personal and family problem. Over $88 \%$ conceptualized RAB as a failure of the body's response to $A B$.

There was a relationship between information on $A D R$ and schooling; knowledge about $A B$ effect on autochthonous flora; adequacy of $A B$ to cold, influenza and sore throat; the suspected need for $A B$ by the patient; the decision to initiate $A B$ by the physician and confidence in the non-prescribing physician $(p<0.05)$.

Conclusions: In general, the patients showed low knowledge in several areas, although it is overlapping with the literature. In this way, the family doctor can direct his actions to adequate information about the concept of RAB and implications in the ecosystem.

Keywords: Bacterial drug resistance; Anti-bacterial agents; Infection control; Health literacy. 


\section{ANEXO I \\ Questionário}

\section{Introdução}

O uso incorreto de antibióticos é cada vez mais comum a nível mundial e está relacionado com o aparecimento de resistência a determinados antibióticos. A falta de informação na população geral relativamente a este tema é uma falha constante e que deve ser corrigida com urgência.

A realização deste trabalho tem como objetivo caracterizar a perceção sobre a utilização de antibióticos em indivíduos com idades compreendidas entre os 18 e 69 anos.

\section{PARTE I.}

\section{Género}

\section{Masculino}

Idade
$18-24$ anos
$35-44$ anos
$55-64$ anos

\section{Escolaridade}

$1^{\circ}$ ao 4 ano

$7^{\circ}$ ao $9^{\circ}$ ano

Mais de $12^{\circ}$ ano

\section{PARTE II.}

1. Quando foi a última vez que tomou um antibiótico?
a. Há menos de 6 meses
b. Há mais de 6 meses e menos de 1 ano
c. Há mais de 1 ano
d. Nunca
e. Não me recordo

2. Assinale com um $X$ em "Concordo", "Não sei" ou "Discordo" as seguintes afirmações:

O antibiótico é indicado para tratar infeções causadas por bactérias
Concordo
Não sei
Discordo

O antibiótico é indicado para tratar infeções causadas por vírus
Concordo
$\checkmark$ Não sei
$\square$ Discordo

O antibiótico é semelhante a medicamentos anti-inflamatórios (aspirina, Brufen ${ }^{\circledR}$, etc.)
Concordo
Não sei
Discordo

O antibiótico é indicado para tratar a febre
Não sei
Discordo

Assim, pede-se que preencha o seguinte questionário. Demorará cerca de 5 minutos a preenchê-lo. Este é anónimo e confidencial.

Se já preencheu este questionário previamente, por favor não o faça novamente!

Ao preencher o seguinte questionário aceita participar neste estudo e os dados obtidos serão única e exclusivamente utilizados para este estudo.

Quando terminar, por favor coloque o inquérito na caixa devidamente identificada para o efeito. Obrigado!

3. Assinale com um X em "Concordo", "Não sei" ou "Discordo" as seguintes afirmações:

O antibiótico é um medicamento com efeitos secundários
Concordo
Não sei
Discordo

O antibiótico é um medicamento que pode causar reações alérgicas

$\square$ Concordo $\square$ Não sei $\square$ Discordo

O antibiótico pode matar bactérias «boas» presentes no nosso corpo (pele, intestino)
Concordo
Não sei
Discordo

4. Assinale com um $X$ em "Concordo", "Não sei" ou "Discordo" as seguintes afirmações:

Guardar em casa o «resto» de antibiótico do tratamento anterior para usar depois

$\square$ Concordo $\square$ Não sei $\square$ Discordo Utilizar um antibiótico de familiar/amigo se os meus sintomas são iguais
Concordo
Não sei
Discordo

Se os meus sintomas foram tratados com antibiótico no passado será igual no futuro
Concordo
Não sei
Discordo

5. Em qual das seguintes situações o antibiótico é indicado? (pode assinalar mais que 1)
a. Constipação
b. Gripe
c. Bronquite aguda
d. Pneumonia
e. Diarreia há mais de 7 dias
f. Dor de garganta há mais de 5 dias
g. Malária
h. Sida

6. Assinale com um X em "Concordo", "Não sei" ou "Discordo" as seguintes afirmações:

Normalmente sou capaz de suspeitar quando preciso de tratamento com antibiótico
Concordo
Não sei
Discordo 
Já fiz tratamento com antibiótico por iniciativa própria

\section{$\square$ Concordo $\square$ Não sei $\square$ Discordo}

A decisão de iniciar antibiótico deve ser tomada pelo médico

$\square$ Concordo $\square$ Não sei $\square$ Discordo

Tenho confiança no médico se não receitar antibiótico

$\square$ Concordo $\square$ Não sei $\square$ Discordo

Tenho confiança no médico que receitar antibiótico

$\square$ Concordo $\square$ Não sei $\square$ Discordo

7. Assinale com um X em "Concordo", "Não sei" ou

"Discordo" as seguintes afirmações:

A lavagem das mãos ao longo do dia evita a contaminação com bactérias

\section{Concordo $\quad \square$ Não sei $\quad \square$ Discordo}

A lavagem das mãos antes de abandonar o Centro de Saúde previne infeções futuras

$\square$ Concordo $\square$ Não sei $\square$ Discordo

Em caso de tosse, deve-se tossir para o braço

$\square$ Concordo $\square$ Não sei $\square$ Discordo

Em caso de contacto com secreções respiratórias

(expetoração, ranho) deve-se lavar as mãos de seguida

$\square$ Concordo $\square$ Não sei $\square$ Discordo

A ventilação adequada (casa, local de trabalho, etc.) evita o contágio com bactérias
Concordo
Não sei
Discordo

8. Em algum momento ouviu falar em "Resistência a antibióticos"?
a. Sim
b. Não

- CASO a sua resposta seja "Não" a sua participação termina aqui.

- CASO a sua resposta seja "Sim" siga para a pergunta 9.1).

8.1 Se respondeu "Sim" na pergunta anterior, como foi informado?
a. Médico
b. Enfermeiro
c. Farmacêutico
d. Familiar/amigo
e. Meios de comunicação social
f. Campanha em saúde
g. Não me recordo

8.2 Assinale com um X em "Concordo", "Não sei" ou "Discordo" as seguintes afirmações:

A resistência a antibióticos dá-se quando o nosso corpo já não responde ao antibiótico
Concordo
Não sei
Discordo

A resistência a antibióticos dá-se quando uma bactéria perde a sensibilidade natural a um ou mais antibióticos
Concordo
Não sei
Discordo

As bactérias resistentes a antibióticos transmitem-se de pessoa para pessoa

$\square$ Concordo $\square$ Não sei $\square$ Discordo

As infeções por bactérias resistentes são difíceis e, por vezes, impossíveis de tratar
Concordo
Não sei
Discordo

8.3 Em algum momento recusou/adiou a toma de antibiótico por medo da resistência a antibióticos?
a. Sim
b. Não

8.4 Assinale com um X em "Concordo", "Não sei" ou "Discordo" as seguintes afirmações:

A resistência a antibióticos é um problema somente nos hospitais
Concordo
Não sei
Discordo

A resistência a antibióticos é um problema para mim e para a minha família
Concordo
Não sei
Discordo

A resistência a antibióticos é um problema mundial e uma causa de morte cada vez mais comum
Concordo
Não sei
Discordo

8.5 Assinale com um $X$ as acções que podem ser benéficas no combate à resistência a antibióticos? (pode assinalar mais que 1)

a. Diminuir o uso de herbicidas na agricultura

b. Maior rigor no uso de antibióticos pelo(a) veterinário(a)

c. Criação de novos antibióticos

d. Atualização do plano nacional de vacinação

e. Interromper o tratamento quando houver melhoria dos sintomas

8.6 Assinale com um X em "Concordo", "Não sei" ou "Discordo" as seguintes afirmações:

A resistência a antibióticos resulta do uso incorreto dos antibióticos

Concordo $\quad \square$ Não sei $\quad \square$ Discordo Um antibiótico utilizado em situações desnecessárias numa região (e.g., Maia) pode perder a eficácia e não ser útil para os seus cidadãos

\section{Concordo $\quad \square$ Não sei $\quad \square$ Discordo}

O cumprimento das indicações do médico em como tomar o antibiótico (dose, hora e número de dias) é uma forma de prevenir a resistência a antibióticos
Concordo
Não sei
Discordo

O facto de o médico receitar o antibiótico mais tarde é uma forma de prevenir a resistência a antibióticos
Concordo
Não sei
Discordo 